\title{
A STUDY OF MALPOSITION OF CENTRAL VENOUS CATHETER - A COMPARATIVE STUDY BETWEEN ULTRASOUND GUIDED AND ANATOMICAL LANDMARK TECHNIQUE
}

\author{
Rajbanshi $L K^{1^{*}}$, Karki $S B^{2}$, Ariyal $B^{1}$
}

\section{Affiliation}

1. Lecturer, Departmet of Anaesthesiology and Critical Care, Birat Medical College and Teaching Hospital

2. Consultant Anaesthesiologist, Departmet of Anaesthesiology and Critical Care, Birat Medical College and Teaching Hospitall

\section{ARTICLE INFO}

Article History
Received : 15 October, 2017
Accepted : 26 October, 2017
Published : 31 December, 2017

(C) Authors retain copyright and grant the journal right of first publication with the work simultaneously licensed under Creative Commons Attribution License CC - BY 4.0 that allows others to share the work with an acknowledgment of the work's authorship and initial publication in this journal.

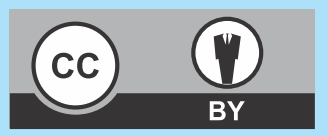

ORA 43

DOI: $\underline{\text { http://dx.doi.org/10.3126/bjhs.v2i3.18943 }}$

\author{
* Corresponding Author \\ Dr Lalit Kumar Rajbanshi \\ Lecturer \\ Departmet of Anaesthesiology and Critical Care \\ Birat Medical College and Teaching Hospital \\ Email: Ialitrajbanshi@gmail.com
}

\section{Citation}

Rajbanshi LK, Karki SB, Ariyal B. A Study of Malposition of Central Venous Catheter - A Comparative Study Between Ultrasound Guided and Anatomical Landmark Technique. BJHS 2017;2(3)4 : 277-281

\begin{abstract}
Introduction

Central venous catheterization is a routine procedure for long-term infusion therapy and central venous pressure measurement. Sometimes, the catheter tip may be unintentionally placed at the position other than the junction of superior vena cava and right atrium. This is called malposition and can lead to erroneous pressure measurement, increase risk of thrombosis, venous obstruction or other life threatening complications like pneumothorax, cardiac temponade.
\end{abstract}

\section{Objectives}

This study aimed to observe the incidence of the malposition and compare the same between ultrasound guided catheterization and blind anatomical landmark technique.

\section{Methodology}

This study was a prospective comparative study conducted at the intensive care unit of Birat Medical College and Teaching Hospital for two-year duration. All the catheterizations were done either with the use of real time ultrasound or blind anatomical landmark technique. The total numbers of central venous catheterization, the total incidences of malposition were observed. Finally the incidences were compared between real time ultrasound guided technique and blind anatomical landmark technique.

\section{Results}

In two-year duration of the study, a total of 422 central venous cannulations were successfully done. The real time ultrasound was used for 280 cannulations while blind anatomical landmark technique was used for 162 patients. The study observed various malposition in 36 cases (8.5\%). The most common malposition was observed for subclavian vein to ipsilateral internal jugular vein (33.3\%) followed by subclavian to subclavian vein $(27.8 \%)$ and internal jugular to ipsilateral subclavian vein (16.7\%). In four patients the catheter had a reverse course in the internal jugular vein while the tip was placed in pleural cavity in three cannulations. There was coiling of the catheter inside left subclavian vein in one patient. The malposition was significantly reduced with the use of the real time ultrasound $(P<0.001)$. However there is no significant difference in the incidence of the various malposition between ultrasound guidance technique and blind anatomical landmark technique when compared individually.

\section{Conclusion}

The malposition of the central venous catheter tip was common complication with the overall incidence of $8.5 \%$. The most common malposition was subclavian vein to internal jugular vein. The use of real time ultrasound during the catheterization procedure can significantly reduced the risk of malposition.

\section{KEY WORDS}

Central venous catheter; malposition; ultrasound; superior venacava. 


\section{INTRODUCTION}

Central venous catheter insertion is a common invasive procedure indicated for fluid resuscitation, inotrope infusion, blood transfusion, chemotherapy and administration of various drugs. ${ }^{1}$ The most commonly used centrally placed veins for the cannulation are subclavian, internal jugular and femoral veins. The catheter is inserted by threading the catheter over the guide wire, a technique called seldinger technique. The catheter is placed at the right atrium with the tip placed at the junction of superior vena cava and right atrium. On chest radiograph, the tip of the catheter should lie at $2 \mathrm{~cm}$ proximal to the pericardial line. ${ }^{2}$ Optimal placement of central venous catheters (CVC) is essential for accurate monitoring of central venous pressure (CVP) in major surgeries and ensuring long-term use of the catheter for managing the critically ill patient.

Insertion of the catheter in to the right atrium can be accomplished either by blind anatomical landmark technique or by the ultrasound guided real time cannulation technique. Technical expertise along with awareness of the potential complications and a sound knowledge of the anatomy of the veins are essential for the successful central venous cannulations. ${ }^{3}$ However, complications like pneumothorax, haematoma, thrombosis, nerve injury, arterial puncture, and malposition are unavoidable in some cases even with the skillful hand and appropriate anatomical landmark technique. ${ }^{4}$ Catheter tip malposition is one of such complications which can lead to the erroneous measurement of the central venous pressure and can predispose to the risk of venous obstruction, thrombosis, clotting or even erosion of the venous wall. ${ }^{5}$ The malposition of catheter tip can be suspected when there is erroneous CVP value or suboptimal waveform pattern which can be confirmed by the chest radiography and real time ultrasound. ${ }^{6}$ The misplacement of the catheter tip can occur at the time of insertion with the guide wire going into the wrong direction or later on due to the migration of the catheter tip due to the inappropriate movement or intra thoracic pressure changes induced by coughing, sneezing or straining. The various factors like position of the head, force applied for threading the guide wire and catheter, the caliber of the vein can be responsible for the inappropriate position of the tip. ${ }^{5}$

Incidence of malposition of central venous catheterization (CVC) ranges from 1-60\%. ${ }^{7}$ The catheter tip can be directed to the ipsilateral internal jugular vein from subclavian vein and vice versa or towards the contralateral subclavian vein. Rarely, the catheter can take a U turn with the reversal of the direction. There are many literatures mainly case reports on the various malposition of the catheter but however none of the literatures has actually measured the burden of the malposition during central venous cannulation.

Thus, this study has put an effort to evaluate the incidence of the possible malposition of the central venous catheter and comparing the incidence between ultrasound guided and anatomical landmark technique.

\section{METHODOLOGY}

This study was prospective observational and comparative study conducted at department of intensive care medicine of Birat Medical College and Teaching Hospital. Ethical approval was taken from the institutional review committee and written consent was taken from the close patient relatives. The study duration was two years starting from January 2015 to January 2017.

The consultant anesthesiologist did all the central venous cannulations. The choice of whether to use real-time ultrasound or anatomical land mark technique was left to the decision of the operator and subjected to the availability of the ultrasound at that particular time in ICU.

The study used portable ultrasound (My Sono U6, Samsung Medison) with linear probe having frequency 5-12 HZ. Insertion was performed aseptically covering the probe with sterile probe cover. Similarly the universal precaution for sterility was strictly followed for landmark technique.

A chest radiograph was done immediately after the completion of the procedure in order to confirm the correct position of the catheter or other associated complications.

The total number of the central venous catheter inserted in two-year durations was included in the study. Central venous catheter insertion requiring more than two attempts, insertion done by the junior duty doctors was excluded from the study. The use of real time ultrasound and blind anatomical landmark were also noted for the comparison. The study analyzed one of major complications of the central venous cannulation i.e. malposition of the central venous catheter which was confirmed by the chest radiograph along with the absence of the free flow of the blood from any of the lumen. The following were the expected malpositions in the study

- Internal jugular vein to ipsilateral subclavien vein and vice versa

- One subclavien vein to the contralateral subclavian vein and vice versa

- Reversal of the direction

- Pleural puncture

The study observed the overall incidence of the malposition out of total central venous catheterization, the type of most common malposition and finally compared the incidence of the different type of malposition between real time ultrasound and anatomical landmark technique.

The collected data were compiled in windows excel and results were analyzed by means of SPSS 17, a software designed for quantitative data analysis.

Parametric continuous data were expressed as mean with standard deviation while categorical data were expressed as percentage (\%). Independent sample t test was used for continuous parametric data for the comparison of mean and Chi-square test was used to compare incidences of the malposition between the real time ultrasound and blind anatomical landmark technique. A P-value less than 0.05 was taken as significant. 


\section{RESULTS}

A total of 422 successful central venous cannulations were done in the study duration. Real time ultrasound was used for $280(66.4 \%)$ cannulations while blind anatomical landmark technique was used for $142(33.6 \%)$ cannulations. Malposition of the catheter was observed only in 36 cases that accounts to the incidence of $8.5 \%$ in total.

The demographic profile of the patients having malposition was non significant as shown in table 1.

\begin{tabular}{|c|c|c|c|c|c|}
\hline & & USG guided & $\begin{array}{l}\text { Anatomical } \\
\text { landmark }\end{array}$ & P Value & Total \\
\hline \multicolumn{2}{|c|}{ Age (years) } & $35.85 \pm 11.70$ & $41.22 \pm 13.73$ & 0.565 & \\
\hline \multicolumn{2}{|c|}{ Weight (kg) } & $60.77 \pm 17.12$ & $62.96 \pm 9.30$ & 0.620 & \\
\hline \multicolumn{2}{|c|}{ BMI $\left(\mathrm{kg} / \mathrm{m}^{2}\right)$} & $22.86 \pm 7.24$ & $25.14 \pm 8.92$ & 0.681 & \\
\hline \multirow{2}{*}{ Sex } & Male & $8(22.2 \%)$ & $\begin{array}{c}16 \\
(44.4 \%)\end{array}$ & & $24(66.6 \%)$ \\
\hline & Female & $5(13.8 \%)$ & $\begin{array}{c}7 \\
(19.4 \%)\end{array}$ & & $12(33.2 \%)$ \\
\hline
\end{tabular}

Malposition of the catheter was observed in 13 (4.6\%) cannulations with the real time ultrasound guided technique while blind anatomical landmark technique had $23(16.2 \%)$ cases with malposition of the catheter (Table 2). There was a strong association of the incidences of malposition between USG and blind procedure $(P<0.001)$. The incidence of malposition among blind procedure was found to be almost 4 times more among USG procedure (16.2\% Vs. 4.6\%).

Table 2: Comparison of malposition betwen USG guided and anatomical landmark group.

\begin{tabular}{lllll}
\hline Malposition & $\begin{array}{c}\text { USG } \\
\text { guided }\end{array}$ & $\begin{array}{l}\text { Anatomical } \\
\text { landmark }\end{array}$ & Total & P value \\
Yes & $13(4.6 \%)$ & $23(16.2 \%)$ & $36(8.5 \%)$ & 0.0001 \\
\hline No & $267(95.4 \%)$ & $119(83.8 \%)$ & $386(91.5 \%)$ & \\
\hline Total & $280(66.4 \%)$ & $142(33.6 \%)$ & 422 &
\end{tabular}

As shown in table no 3 , the most common type of malposition observed in the study was found during subclavian vein cannulation with the catheter tip lying at ipsilateral internal jugular vein (subclavian to internal jugular, 33.3\%). This was followed by right subclavian vein to left subclavian vein and vice versa (10 cases, $27.8 \%$ ). The incidence of the malposition from internal jugular vein to the ipsilateral subclavian vein was $16.7 \%$. Similarly, there was reversal of the direction of the catheter in the internal jugular vein in 4 cases $(11.1 \%)$. In three cases $(8.3 \%)$, the catheter was inadvertently placed in the pleural space. All these three malposition occurred with the blind anatomical landmark technique during internal jugular vein cannulation. As the infusion was not started and the malposition was immediately detected by the absence of blood withdraw and immediate chest $x$ ray, the unwanted complications like pneumothorax, pleural effusion were avoided in these cases. In one case there was coiling of the catheter inside the left subclavien vein but the tip was correctly placed at the right atrium.
However, the differences of proportions based on different types of malposition in between two groups were non significant $(P>0.05)$ (Table 3).

Table 3: Comparison of individual malposition between USG guided and anatomical landmark technique.

\begin{tabular}{lrrrr}
\hline Malposition & $\begin{array}{c}\text { USG } \\
\text { guided }\end{array}$ & $\begin{array}{c}\text { Anatomical } \\
\text { landmark }\end{array}$ & Total & P value \\
$\begin{array}{l}\text { Subclavian to Internal } \\
\text { Jugular vein }\end{array}$ & $5(38.4 \%)$ & $7(30.5 \%)$ & $12(33.3 \%)$ & 0.902 \\
\hline $\begin{array}{l}\text { Subclavian to } \\
\text { subclavian and vice } \\
\text { versa }\end{array}$ & $4(30.8 \%)$ & $6(26.1 \%)$ & $10(27.8 \%)$ & 0.931 \\
\hline $\begin{array}{l}\text { Internal Jugular to } \\
\text { Subclavian }\end{array}$ & $2(15.4 \%)$ & $4(17.4 \%)$ & $6(16.7 \%)$ & 0.756 \\
\hline Reversal of direction & $1(7.7 \%)$ & $3(13.0 \%)$ & $4(11.1 \%)$ & 0.951 \\
\hline Pleural Space & $0(0.0 \%)$ & $3(13.0 \%)$ & $3(8.3 \%)$ & NA \\
\hline Coiling & $1(7.7 \%)$ & $0(0.0 \%)$ & $1(2.8 \% \%)$ & NA \\
\hline Total & 13 & 23 & 36 & \\
\hline
\end{tabular}

\section{DISCUSSION}

Central venous catheter (CVC) malposition is one of the major complications of the procedure. The malposition can lead to serious life threatening complications like bleeding, pneumothorax and pleural effusion if not detected and managed immediately. Moreover, malposition of a CVC can lead to inaccurate measurement of the central venous pressure with abnormal waveform and can be unsuitable for the infusion of various vasoactive drugs and other situations requiring long-term infusions. ${ }^{8}$

The present study was conducted to evaluate the incidence of the central venous catheter malposition, the most common type of malposition and comparison of the catheter malposition between ultrasound guided technique and blind anatomical landmark technique. As observed in the study $36(8.5 \%)$ out of 422 total cannulations in two years duration were found to have catheter tip malposition. This incidence was with in the range of the incidences of malposition reported in various literatures report. ${ }^{7}$

The internal jugular vein and the subclavian vein on either side unite to form right and left brachiocephalic vein which in turn drains in to the superior venacana. The hemiazygous vein and the thoracic duct drains into the left brachiocephalic vein while azygous vein drains in to the superior venacava. The internal mammary vein, which travels along the border, also drains into the brachiocephalic vein. Because of these free communication and close proximity of the various small veins draining in to the central vein, there is high possibility of the catheter passing into any of the inadvertent position.

It is more convenient to cannulate the right internal jugular vein as this provides a direct and straight pathway to the right atrium that decreases the possibility of malposition. Similarly, the superior venacava is in close vicinity of the mediastinum making it more prone for perforation with the guide wire, dilator or catheter and leading to the malposition and other life threatening complications. ${ }^{9,10}$ 
The study observed that the most common catheter malposition was during subclavian vein cannulation with the catheter passing into the ipsilateral internal jugular vein (33.3\%). This was followed by catheter passing from one subclavian vein to the contralateral subclavian vein and vice versa $(27.8 \%)$. The possible reason for these malpositions could be the change in the direction of the J tip of the guide wire during the procedure. ${ }^{11}$ The malposition can be minimized by constantly keeping the J tip of the guide wire in a caudal direction. Besides this, excessive force applied during threading of the guide wire or catheter and excessive length of the guide wire insertion can be the possible reason for misplacement of the catheter tip. The optimal length of the guide wire that can be inserted with the least possibility of malposition was suggested to be 18 to $20 \mathrm{~cm}$. ${ }^{12,13}$

Position of the head and shoulder can sometimes determine the direction of the guide wire and the catheter subsequently. The angle between subclavian vein and ipsilateral internal jugular vein is usually acute in neutral head position. The angle is increased making the two veins more in line when the head is turned away from the needle puncture. This increases the risk of catheter passing in to the internal jugular vein and vice versa. ${ }^{14,15}$ in the same way the angle between the subclavien vein and innominate vein is reduced by lowering the shoulder position and this increases the risk of malposition. ${ }^{16}$ Another effective method for minimizing the malposition from subclavian to internal jugular vein is to apply external pressure on IJV during the threading of the guide wire.

In $16.7 \%$ of the total catheter malposition, the catheter tip was located into the ipsilateral subclavien vein from the internal jugular vein. Again the possible reason for this malposition could be the inappropriate direction of the $J$ tip of guide wire, excessive length of the guide wire or inappropriate position of the head $n$ shoulder.

The catheter took a reverse course in $11 \%$ (4 cases) during internal jugular vein cannulation. In one patient the catheter was found in the ipsilateral external jugular vein, which was confirmed by chest x-ray and ultrasound. Any obstruction due to thrombosis, stenosis distal to the opening of internal jugular vein in the brachiocephalic vein or the wrongly directed tip of guide wire can be the possible reasons for this malposition.

The catheter tip lying in the pleural space can be a rare but life threatening complications that was observed in $8.3 \%$ of total malposition.

The right border of SVC, azygos, hemiazygos, and internal thoracic veins are immediately adjacent to the pleura. We assumed the excessive force during guide wire or catheter advancement and partial or complete obstruction of the superior venacava can lead to erosion of the venous wall and guide wire or catheter can be wrongly inserted into the pleural cavity. The catheter malpositions were immediately diagnosed with the chest $x$ ray in all the three cases and subsequently removed. Fortunately none of the cases developed any complication associated with the pleural puncture.
In one case, the catheter had coiling inside the left subclavian vein but the tip was at the superior venacava. The valve present in the left subclavian vein might have obstructed the guide wire and made it coiled and later on the catheter following the same course. The catheter was left in situ without any manipulation as the blood flow was good and catheter tip was placed in SVC.

The study compared the incidences of malposition between the real-time ultrasound guided technique and blind anatomical approach. There was statistically significant reduction $(4.6 \%$ vs. $16.2 \%$, $\mathrm{P}$ value $<0.001)$ in the incidence of malposition with the use of ultrasound. However the individual comparison of different malpositioins between the two groups didn't show much difference.

Real-time ultrasound is routinely used for the cannulation of the central veins as it provides a real-time image of the veins, artery and other surrounding soft tissues. Moreover, the passage of guide wire inside the vein can easily be detected thus decreasing the possibility of malposition. The real time ultrasound is helpful especially in older patient or in patient with shock where the veins are collapsed. The use of ultrasound has significantly decreased the incidences of malposition. ${ }^{17}$

However, the other factors like head position, direction of the tip of the J point of guide wire, the force applied during threading of the guide wire and presence of any obstruction play a vital role for the misplacement of the catheter tip.

\section{LIMITATION OF THE STUDY}

The number of the cases for the comparison between the two groups could not be equalized because of the operator convenience and the technical difficulty in localizing the vein during cannulation. The comparable samples would have produced much more reliable result. The single centered study could not detect the other rare malpositions like mediastinal puncture, pericardial rupture, and intrathecal placement.

\section{RECOMMENDATIONS}

The study recommends the use of real time ultrasound for the cannulation procedure to minimize the complications. The study strongly supports the fact that the real time sonology of needle, guide wire and catheter insertion and its ability to locate the catheter tip can detect the malposition at the earliest and prevent the unwanted complications. Similarly, technical expertise of the procedure, thorough knowledge of normal anatomy and anatomical variations of the veins are equally important to decrease the risk of malposition

\section{CONCLUSION}

Central venous catheter malposition is one of the major complications and can lead to life threatening conditions if not detected and managed immediately. The study had observed a over all incidence of $8.3 \%$ malposition. Catheter passing into internal jugular vein from subclavian vein was the most common malposition. The use of real-time 
ultrasound has significantly decreased the incidence of the malposition. The direction of the J tip of guide wire, length of the guide wire, position of the head and shoulder as well as caliber of the vein can be responsible for the malposition of the catheter tip.

\section{ACKNOWLEDGEMENT}

The author would like to thank all the staffs in the intensive care unit of Birat Medical College and Teaching Hospital who directly or indirectly helped to complete this study. The author sincerely acknowledged Dr Surya Niraula, professor, department of community medicine, BPKIHS, for his contribution in statistical analysis of the study.

\section{CONFLICT OF INTEREST}

None

\section{REFERENCES}

1. Tan PL, Gibson M. Central venous catheters: The role of radiology. Clin Radiol. 2006;61:13-22

2. Coskun D, Mahli A, Oncul S, Ilvan G, Dalgic A. Malposition of subclavian vein catheter inserted through indirect technique in a pediatric liver transplantation: A case report. Cases J 2009;2:7998

3. Shannon D. Central venous catheter-induced pericardial effusion in a neonate: A case study and recommendations for practice. Neonatal Netw. 2014;33:341-8.

4. de Jonge RC, Polderman KH, Gemke RJ. Central venous catheter use in the pediatric patient: Mechanical and infectious complications. Pediatr Crit Care Med 2005;6:329-39

5. Collier PE, Blocker SH, Graff DM, Doyle P. Cardiac tamponade from central venous catheters. Am J Surg 1998; 176: 212-4

6. Agrawal P, Gupta B, D'souza N. Coiled central venous catheter in superior vena cava. Indian J Anaesth 2010;54:351-2

7. Malatinský J, Kadlic T, Májek M, Sámel M. Misplacement and loop formation of central venous catheters. Acta Anaesthesiol Scand. 1976;20:237-47.

8. Hofmann-Preiss K, Becker A, Sailer S. Radiologic and clinical followup of central venous indwelling catheters in home parenteral nutrition. Infusion Stherapie. 1991;18:292-5

9. Gibson F, Bodenham A. Misplaced central venous catheters: Applied anatomy and practical management. Br J Anaesth. 2013;110:333-46.

10. Bannon MP, Heller SF, Rivera M. Anatomic considerations for central venous cannulation. Risk Manag Healthc Policy. 2011;4:27-39.
11. Tripathi M, Dubey PK, Ambesh SP. Direction of the J-tip of the guidewire, in seldinger technique, is a significant factor in misplacement of subclavian vein catheter: a randomized, controlled study. Anesth Analg 2005;100:21-4.

12. Andrews RT, Bova DA, Venbrux AC. How much guidewire is too much? Direct measurement of the distance from subclavian and internal jugular vein access sites to the superior vena cava-atrial junction during central venous catheter placement. Crit Care Med 2000;28:138-42.

13. McGee WT, Ackerman BL, Rouben LR, Prasad VM, BandiV, Mallory $\mathrm{DL}$. Accurate placement of central venous catheters: a prospective, randomized, multicenter trial. Crit Care Med 1993;21:1118-23.

14. Jung CW, Bahk JH, Kim MW, Lee KH, Ko H. Head position for facilitating the superior vena caval placement of catheters during right subclavian approach in children. Crit Care Med 2002;30:297-299.

15. Boon JM, van Schoor AN, Abrahams PH, Meiring JH, Welch T, Shanahan D. Central venous catheterization - an anatomical review of a clinical skill - part1: subclavian vein via the infraclavicular approach. Clin Anat 2007;20:602-611

16. Kitagawa N, Oda M, Totoki T, Miyazaki N, Nagasawa I, Nakazono T, Tamai T, Morimoto M. Proper shoulder position for subclavian venipuncture: a prospective randomized clinical trial and anatomical perspectives using multislice computed tomography. Anesthesiology 2004;101:1306-10

17. Massimo Cajozzo, Gerlando Cocchiara, Geo Greco, Roberto Vaglica, et al: J. Surg. Oncol.2004;88:267-268. 